\title{
Research Article \\ Generalized Fuzzy Interior Ideals in Abel Grassmann's Groupoids
}

\author{
Asghar Khan, ${ }^{1}$ Young Bae Jun, ${ }^{2}$ and Tahir Mahmood ${ }^{3}$ \\ ${ }^{1}$ Department of Mathematics, COMSATS Institute of Information Technology, 22060 Abbottabad, Pakistan \\ ${ }^{2}$ Department of Mathematics Education and RINS, Gyeongsang National University, \\ Chinju 660-701, South Korea \\ ${ }^{3}$ Department of Applied Mathematics, International Islamic University, 45320 Islamabad, Pakistan
}

Correspondence should be addressed to Asghar Khan, azhar4set@yahoo.com

Received 27 August 2009; Accepted 24 February 2010

Academic Editor: Peter Basarab-Horwath

Copyright (C) 2010 Asghar Khan et al. This is an open access article distributed under the Creative Commons Attribution License, which permits unrestricted use, distribution, and reproduction in any medium, provided the original work is properly cited.

Using the notion of a fuzzy point and its belongness to and quasicoincidence with a fuzzy subset, some new concepts of a fuzzy interior ideal in Abel Grassmann's groupoids $S$ are introduced and their interrelations and related properties are invesitigated. We also introduce the notion of a strongly belongness and strongly quasicoincidence of a fuzzy point with a fuzzy subset and characterize fuzzy interior ideals of $S$ in terms of these relations.

\section{Introduction}

The idea of a quasicoincidence of a fuzzy point with a fuzzy set, which is mentioned in $[1,2]$, played a vital role to generate some different types of fuzzy subgroups. It is worth pointing out that Bhakat and Das [2] gave the concepts of $(\alpha, \beta)$-fuzzy subgroups by using the "belongs to" relation $(\in)$ and "quasicoincident with" relation $(q)$ between a fuzzy point and a fuzzy subgroup, and they introduced the concept of an $(\in, \in \vee q)$-fuzzy subgroup. In particular, $(\epsilon, \in \vee q)$-fuzzy subgroup is an important and useful generalization of Rosenfeld's fuzzy subgroup. It is now natural to investigate similar type of generalizations of the existing fuzzy subsystems of other algebraic structures. With this objective in view, Davvaz $[3,4]$ introduced the concept of $(\in, \in \vee q)$-fuzzy sub-near-rings (R-subgroups, ideals) of a near-ring and investigated some of their interesting properties. Jun and Song [5] discussed general forms of fuzzy interior ideals in semigroups. Kazanci and Yamak introduced the concept of a generalized fuzzy bi-ideal [6]. Also Davvaz and many others used this concept in several other algebraic structures (see [7-16]). Jun [13, 17], gave the concept of $(\alpha, \beta)$ fuzzy subalgebra of a BCK/BCI-algebras. In [18], Luo introduced the concept of a strong neighborhood. According to him, a fuzzy point $x_{\lambda}(0<\lambda<1)$ is said to be strongly belong to 
a fuzzy subset $F$, denoted by $x_{\curlywedge} \in F$, if and only if $F(x)>\lambda$. $\lambda$-strong cut set $F_{\underline{\lambda}}$ of $F$ is given by $F_{\underline{\lambda}}=\{x \in X \mid F(x)>\lambda\}$, where $X$ is a nonempty set. The idea of $Q$-neighborhood in fuzzy topology was introduced by $\mathrm{Pu}$ and Liu in [19]. According to them, a fuzzy point $x_{\curlywedge}$ is said to be strongly quasicoincident with $F$, denoted by $x_{\lambda} q F$, if and only if $\lambda+F(x)>1$.

An Abel Grassmann's groupoid, abbreviated as AG-groupoid, is a groupoid $S$ whose elements satisfy the left invertive law: $(a b) c=(c b) a$ for all $a, b, c \in S$. An AG-groupoid is the midway structure between a commutative semigroup and a groupoid. It is a useful nonassociative structure with wide applications in theory of flocks. In an AG-groupoid the medial law, $(a b)(c d)=(a c)(b d)$ for all $a, b, c \in S$ (see [20]). If there exists an element $e$ in an AGgroupoid $S$ such that $e x=x$ for all $x \in S$ then $S$ is called an AG-groupoid with left identity $e$. If an $A G$-groupoid $S$ has the right identity then $S$ is a commutative monoid. If an AG-groupoid $S$ contains left identity then $(a b)(c d)=(d c)(b a)$ holds for all $a, b, c \in S$. Also $a(b c)=b(a c)$ holds for all $a, b, c \in S$.

In this paper, we define $(\alpha, \beta)$-fuzzy interior ideals of an $A G$-groupoid and give some interesting characterizations of an $A G$-groupoids in terms of $(\alpha, \beta)$-fuzzy interior ideals. We also introduce the notion of $(\underline{\alpha}, \underline{\beta})$-fuzzy interior ideals of an $A G$-groupoid.

\section{Preliminaries}

For subsets $A, B$ of an $A G$-groupoid $S$, we denote $A B=\{a b \in S \mid a \in A, b \in B\}$. A nonempty subset $A$ of an $A G$-groupoid $S$ is called an $A G$-subgroupoid of $S$ if $A^{2} \subseteq A$. $A$ is called an interior ideal of $S$ if $(S A) S \subseteq A$.

Let $S$ be an $A G$-groupoid. By a fuzzy subset $F$ of $S$, we mean a mapping, $F: S \rightarrow[0,1]$.

For fuzzy subsets $F_{1}$ and $F_{2}$ of $S$, define

$$
\begin{aligned}
F_{1} \circ F_{2}: S \longrightarrow[0,1], a \longmapsto & F_{1} \circ F_{2}(a) \\
& = \begin{cases}\bigvee_{a=y z} \min \left\{F_{1}(y), F_{2}(z)\right\}, & \text { if } a=y z(\forall a, x, y \in S), \\
0, & \text { if } a \neq y z .\end{cases}
\end{aligned}
$$

We denote by $\mathcal{F}(S)$ the set of all fuzzy subsets of $S$. One can easily see that $(\mathcal{F}(S), 0)$ becomes an AG-groupoid as shown in [21]. The order relation " $\subseteq$ " on $\mathscr{F}(S)$ is defined as follows:

$$
F_{1} \subseteq F_{2} \quad \text { iff } F_{1}(x) \leq F_{2}(x) \quad \forall x \in S, \quad \forall F_{1}, F_{2} \in \mathcal{F}(S)
$$

For a nonempty family of fuzzy subsets $\left\{F_{i}\right\}_{i \in I}$, of an $A G$-groupoid $S$, the fuzzy subsets $\bigcup_{i \in I} F_{i}$ and $\bigcap_{i \in I} F_{i}$ of $S$ are defined as follows:

$$
\begin{aligned}
& \bigcup_{i \in I} F_{i}: G \longrightarrow[0,1], a \longmapsto\left(\bigcup_{i \in I} F_{i}\right)(a):=\sup _{i \in I}\left\{F_{i}(a)\right\}, \\
& \bigcap_{i \in I} F_{i}: G \longrightarrow[0,1], a \longmapsto\left(\bigcap_{i \in I} F_{i}\right)(a):=\inf _{i \in I}\left\{F_{i}(a)\right\} .
\end{aligned}
$$


If $I$ is a finite set, say $I=\{1,2, \ldots, n\}$, then clearly

$$
\begin{aligned}
& \bigcup_{i \in I} F_{i}(a)=\max \left\{F_{1}(a), F_{2}(a), \ldots, F_{n}(a)\right\}, \\
& \bigcap_{i \in I} F_{i}(a)=\min \left\{F_{1}(a), F_{2}(a), \ldots, F_{n}(a)\right\} .
\end{aligned}
$$

Definition 2.1 (cf. [21]). Let $S$ be an AG-groupoid and $F$ a fuzzy subset of $S$. Then $F$ is called a fuzzy interior ideal of $S$, if it satisfies the following conditions.

(B1) (for all $x, y \in S)(F(x y) \geq \min \{F(x), F(y)\})$.

(B2) (for all $x, y, a \in S)(F((x a) y) \geq F(a))$.

Let $F$ be a fuzzy subset of $S$ and $\emptyset \neq A \subseteq S$, then the characteristic function $\chi_{A}$ of $A$ is defined as

$$
\chi_{A}: S \longrightarrow[0,1], a \longmapsto \chi_{A}(a):= \begin{cases}1, & \text { if } a \in A, \\ 0, & \text { if } a \notin A .\end{cases}
$$

Lemma 2.2 (cf. [21]). Let $S$ be an AG-groupoid and $F$ a fuzzy subset of $S$. Then $F$ is a fuzzy interior ideal of $S$ if and only if $x_{A}$ is a fuzzy interior ideal of $S$.

Let $S$ be an AG-groupoid and $F$ a fuzzy subset of $S$. Then for every $\lambda \in(0,1]$ the set

$$
U(F ; \lambda):=\{x \mid x \in S, F(x) \geq \lambda\}
$$

is called a level set of $F$.

The proof of the following lemma is easy and we omit it.

Lemma 2.3. Let $S$ be an AG-groupoid and $F$ a fuzzy subset of $S$. Then $F$ is a fuzzy interior ideal of $S$ if and only if $U(F ; \lambda)(\neq \emptyset)$ is an interior ideal of $S$ for every $\lambda \in(0,1]$.

\section{3. $(\alpha, \beta)$-Fuzzy Interior Ideal}

In what follows let $S$ denote an $A G$-groupoid and let $\alpha, \beta$ denote any one of $\in, q, \in \vee q, \in \wedge q$.

Let $S$ be an $A G$-groupoid and $F$ a fuzzy subset of $S$, then the set of the form

$$
F(y):= \begin{cases}\lambda(\neq 0), & \text { if } y=x \\ 0, & \text { if } y \neq x\end{cases}
$$

is called a fuzzy point with support $x$ and value $\lambda$ and is denoted by $x_{\lambda}$. A fuzzy point $x_{\lambda}$ is said to belong to (resp., quasicoincident with) a fuzzy set $F$, written as $x_{\lambda} \in F$ (resp., $x_{\lambda} q F$ ) if $F(x) \geq \lambda$ (resp., $F(x)+\lambda \geq 1$ ). If $x_{\curlywedge} \in F$ or $x_{\lambda} q F$, then $x_{\lambda} \in \vee q F$. The symbol $\overline{\in \vee q}$ means $\in \vee q$ 
does not hold. A fuzzy point $x_{\curlywedge}$ is said to be strongly belong to (resp., strongly quasicoincident with) a fuzzy set $F$, written as $x_{\lambda} \in F$ (resp., $\left.x_{\lambda} q F\right)$ if $F(x)>\lambda$ (resp., $\lambda+F(x)>1$ ). If $x_{\lambda} \in F$ or $x_{\lambda} \underline{q} F$, then $x_{\lambda} \underline{\in} \vee \underline{q} F$. The symbol $\bar{\epsilon} \vee \underline{q}$ means that $\underline{\in} \vee \underline{q}$ does not hold.

Every fuzzy interior ideal of $\bar{S}$ is an $(\epsilon, \in)$-fuzzy interior ideal of $S$, as shown in the following theorem.

Theorem 3.1. For any fuzzy subset F of $S$. The conditions (B1) and (B2) of Definition 2.1, are equivalent to the following.

(B3) $(\forall x, y \in S)\left(\forall \lambda_{1}, \lambda_{2} \in(0,1]\right)\left(x_{\lambda_{1}} \in F, y_{\lambda_{2}} \in F \rightarrow(x y)_{\min \left\{\lambda_{1}, \lambda_{2}\right\}} \in F\right)$.

(B4) $(\forall x, y, a \in S)(\forall \lambda \in(0,1])\left(a_{\curlywedge} \in F \rightarrow((x a) y)_{\curlywedge} \in F\right)$.

Proof. $(B 1) \rightarrow(B 3)$. Let $x, y \in S$ and $\lambda_{1}, \lambda_{2} \in(0,1]$ be such that $x_{\lambda_{1}} \in F$ and $y_{\lambda_{2}} \in F$. Then $F(x) \geq \lambda_{1}$ and $F(y) \geq \lambda_{2}$. By (B1) we have

$$
F(x y) \geq \min \{F(x), F(y)\} \geq \min \left\{\lambda_{1}, \lambda_{2}\right\},
$$

and so $(x y)_{\min \left\{\lambda_{1}, \lambda_{2}\right\}} \in F$.

$(B 3) \rightarrow(B 1)$. Let $x, y \in S$. Since $x_{F(x)} \in F$ and $y_{F(y)} \in F$. Then by (B3), we have $(x y)_{\min \{F(x), F(y)\}} \in F$ and so $F(x y) \geq \min \{F(x), F(y)\}$.

$(B 2) \rightarrow(B 4)$. Let $x, y, a \in S$ and $\lambda \in(0,1]$ be such that $a_{\curlywedge} \in F$. Then $F(a) \geq \lambda$. By $(B 2)$ we have

$$
F((x a) y) \geq F(a) \geq \lambda,
$$

and so $((x a) y)_{\lambda} \in F$.

$(B 4) \rightarrow(B 2)$. Let $x, y \in S$. Since $a_{F(a)} \in F$, by $(B 4)$, we have $((x a) y)_{F(a)} \in F$ and so $F((x a) y) \geq F(a)$.

\section{4. $(\in, \in \vee q)$-Fuzzy Interior Ideals}

In [5], Jun and Song introduced the concept of a generalized fuzzy interior ideal of a semigroup. In [12], Jun et al. introduced the concept an $(\alpha, \beta)$-fuzzy bi-ideal of an ordered semigroup and characterized ordered semigroups in terms of $(\alpha, \beta)$-fuzzy bi-ideals. In this section we define the notions of $(\in, \in \vee q)$-fuzzy interior ideals of an Abel Grassmann's groupoid and investigate some of their properties in terms of $(\in, \in \vee q)$-fuzzy interior ideals.

Let $F$ be a fuzzy subset of $S$ and $F(x) \leq 0.5$ for all $x \in S$. Let $x \in S$ and $\lambda \in(0,1]$ be such that $x_{\curlywedge} \in \wedge \mathrm{q} F$. Then $x_{\lambda} \in F$ and $x_{\curlywedge} q F$ and so $F(x) \geq \lambda$ and $F(x)+\lambda \geq 1$. It follows that $1<F(x)+\lambda \leq F(x)+F(x)=2 F(x)$, and so $F(x)>0.5$, which is a contradiction. This means that $\left\{x \in S \mid x_{\curlywedge} \in \wedge q F\right\}=\emptyset$.

Definition 4.1. A fuzzy subset $F$ of $S$ is called an $(\alpha, \beta)$-fuzzy interior ideal of $S$, where $\alpha \neq \in \wedge q$, if it satisfies the following conditions:

(B5) $(\forall x, y \in S)\left(\forall \lambda_{1}, \lambda_{2} \in(0,1]\right)\left(x_{\lambda_{1}} \alpha F, y_{\lambda_{2}} \alpha F \rightarrow(x y)_{\min \left\{\lambda_{1}, \lambda_{2}\right\}} \beta F\right)$.

(B6) $(\forall x, y, a \in S)(\forall \lambda \in(0,1])\left(a_{\lambda} \alpha F \rightarrow((x a) y)_{\lambda} \beta F\right)$. 
Proposition 4.2. Let $F$ be a fuzzy subset of $S$. If $\alpha=\in$ and $\beta=\in \vee q$ in Definition 4.1. Then (B5), and (B6), respectively, of Definition 4.1, are equivalent to the following conditions:

(B7) $(\forall x, y \in S)(F(x y) \geq \min \{F(x), F(y), 0.5\})$.

(B8) $(\forall x, y, a \in S)(F((x a) y) \geq \min \{F(a), 0.5\})$.

Remark 4.3. A fuzzy subset $F$ of an $A G$-groupoid $\mathrm{S}$ is an $(\in, \in \mathrm{V} q)$-fuzzy interior ideal of $\mathrm{S}$ if and only if it satisfies conditions (B7), and (B8) of the above proposition.

Using Proposition 4.2, we have the following characterization of $(\in, \in \vee q)$-fuzzy interior ideals of an $A G$-groupoid.

Lemma 4.4. Let $S$ be an $A G$-groupoid and $\emptyset \neq I \subseteq S$. Then $I$ is an interior ideal of $S$ if and only if the characteristic function $X_{I}$ of $I$ is an $(\in, \in \vee q)$-fuzzy interior ideal of $S$.

The converse of Theorem 3.1 is not true in general, as shown in the following example.

Example 4.5. Let $S=\{a, b, c, d, e\}$ be an $A G$-groupoid with the following multiplication:

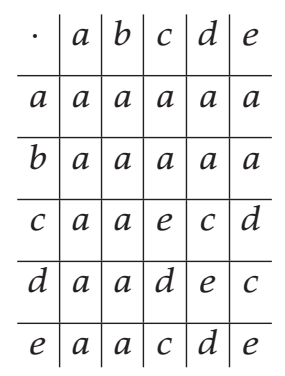

The $(S, \cdot)$ is an $A G$-groupoid. The interior ideals of $S$ are $\{a\}$ and $\{a, c, d, e\}$. Define a fuzzy subset $F: S \rightarrow[0,1]$ by

$$
F(a)=0.8, \quad F(c)=0.6, \quad F(d)=0.4, \quad F(e)=0.2, \quad F(b)=0.1 .
$$

Then

$$
U(F ; \lambda):= \begin{cases}S, & \text { if } \lambda \in(0,0.1], \\ \{a, c, d, e\}, & \text { if } \lambda \in(0.1,0.2], \\ \{a\}, & \text { if } \lambda \in(0.6,1], \\ \emptyset, & \text { if } \lambda \in(0.8,1] .\end{cases}
$$

Obviously, $F$ is an $(\in, \in \vee q)$-fuzzy interior ideal of $S$ by Lemma 4.4. But we have the following.

(i) $F$ is not an $(\in, \in)$-fuzzy interior ideal of $S$, since $d_{0.38} \in F$ but

$$
(d d)_{\min \{0.38,0.38\}}=e_{0.38} \bar{\in} F .
$$


(ii) $F$ is not an $(\in, q)$-fuzzy interior ideal of $S$, since $d_{0.36} \in F$ but

$$
(d d)_{\min \{0.36,0.36\}}=e_{0.36} \bar{q} F \text {. }
$$

(iii) $F$ is not a $(q, \in)$-fuzzy interior ideal of $S$, since $c_{0.52} q F$ and $e_{0.82} q F$ but

$$
(c e)_{\min \{0.52,0.82\}}=d_{0.52} \bar{\epsilon} F \text {. }
$$

(iv) $F$ is not a $(q, \in \vee q)$-fuzzy bi-ideal of $S$, since $c_{0.52} q F$ and $e_{0.82} q F$ but

$$
(c e)_{\min \{0.52,0.82\}}=d_{0.52} \overline{\in \vee q} F .
$$

(v) $F$ is not an $(\in \vee q, \in \wedge q)$-fuzzy interior ideal of $S$, since $d_{0.38} \in \vee q F$ but

$$
(d d)_{\min \{0.38,0.38\}}=e_{0.38} \overline{\in \wedge q} F
$$

(vi) $F$ is not an $(\in \vee q, q)$-fuzzy interior ideal of $S$, since $c_{0.56} \in \vee q F$ and $e_{0.18} \in \vee q F$ but

$$
(c e)_{\min \{0.56,0.18\}}=d_{0.18} \bar{q} F .
$$

(vii) $F$ is not an $(\in \vee q, \in)$-fuzzy interior ideal of $S$, since $d_{0.38} \in \vee q F$, but

$$
(d d)_{\min \{0.38,0.38\}}=d_{0.38} \bar{\in} F \text {. }
$$

(viii) $F$ is not $(\in \wedge q, \in)$-fuzzy interior ideal of $S, d_{0.38} \in \wedge q F$, but

$$
(d d)_{\min \{0.38,0.38\}}=d_{0.38} \bar{\in} F .
$$

(ix) $F$ is not a $(q, q)$-fuzzy interior ideal of $S$, since $c_{0.52} q F$ and $e_{0.82} q F$ but

$$
(c e)_{\min \{0.52,0.82\}}=d_{0.52} \bar{q} F \text {. }
$$


(x) $F$ is not an $(\in, \in \vee q)$-fuzzy interior ideal of $S$, since $c_{0.52} \in F$ and $e_{0.82} \in F$ but

$$
(c e)_{\min \{0.52,0.82\}}=d_{0.52} \overline{\in \vee q} F \text {. }
$$

(xi) $F$ is not an $(\in \vee q, \in \vee q)$-fuzzy interior ideal of $S, c_{0.58} \in F$ and $e_{0.86} \in F$ but

$$
(c e)_{\min \{0.58,0.86\}}=d_{0.58} \overline{\in \vee q} F \text {. }
$$

Remark 4.6. By Remark 4.3, every fuzzy interior ideal of an $A G$-groupoid $S$ is an $(\in, \in \vee q)$ fuzzy interior ideal of $S$. However, the converse is not true, in general.

Example 4.7. Consider the AG-groupoid given in Example 4.5, and define a fuzzy subset $F$ : $S \rightarrow[0,1]$ by

$$
F(a)=0.8, \quad F(c)=0.6, \quad F(d)=0.4, \quad F(e)=0.2, \quad F(b)=0.1 .
$$

Clearly $F$ is an $(\in, \in \vee q)$-fuzzy interior ideal of $S$. But $F$ is not an $(\alpha, \beta)$-fuzzy interior ideal of $S$ as shown in Example 4.5.

Theorem 4.8. Every $(\in, \in)$-fuzzy interior ideal of $S$ is an $(\in, \in \vee q)$-fuzzy interior ideal of $S$.

Proof. It is straightforward.

Theorem 4.9. Every $(\in \vee q, \in \vee q)$-fuzzy interior ideal of $S$ is $(\in, \in \vee q)$-fuzzy interior ideal of $S$.

Proof. Let $F$ be an $(\in \vee q, \in \vee q)$-fuzzy interior ideal of $S$. Let $x, y \in S$ and $\lambda_{1}, \lambda_{2} \in(0,1]$ be such that $x_{\lambda_{1}}, y_{\lambda_{2}} \in F$. Then $x_{\lambda_{1}}, y_{\lambda_{2}} \in \vee q F$, which implies that $(x y)_{\min \left\{\lambda_{1}, \lambda_{2}\right\}} \in \vee q F$. Let $x, y, a \in S$ and $\lambda \in(0,1]$ be such that $a_{\lambda} \in F$. Then $a_{\lambda} \in \vee q F$, and we have $((x a) y)_{\lambda} \in \vee q F$.

Theorem 4.10. Let $F$ be a nonzero $(\alpha, \beta)$-fuzzy interior ideal of $S$. Then the set $F_{0}:=\{x \in S \mid F(x)>$ $0\}$ is an interior ideal of $S$.

Proof. Let $x, y \in F_{0}$. Then $F(x)>0$ and $F(y)>0$. Assume that $F(x y)=0$. If $\alpha \in\{\in, \in \vee q\}$, then $x_{F(x)} \alpha F$ and $y_{F(y)} \alpha F$ but $(x y)_{\min \{F(x), F(y)\}} \bar{\beta} F$ for every $\beta \in\{\in, q, \in \vee q, \in \wedge q\}$, a contradiction. Note that $x_{1} q F$ and $y_{1} q F$ but $(x y)_{\min \{1,1\}}=(x y)_{1} \bar{\beta} F$ for every $\beta \in\{\in, q, \in \vee q, \in \wedge q\}$, a contradiction. Hence $F(x y)>0$, that is, $x y \in F_{0}$. Let $a \in F_{0}$ and $x, y \in S$. Then $F(a)>0$. Assume that $F((x a) y)=0$. If $\alpha \in\{\in, \in \vee q\}$ then, $a_{F(a)} \alpha F$ but $((x a) y)_{F(a)} \bar{\beta} F$ for every $\beta \in\{\in, q, \in \vee q, \in \wedge q\}$, a contradiction. Note that $a_{1} q F$ but $((x a) y)_{\min \{1,1\}}=((x a) y)_{1} \bar{\beta} F$ for every $\beta \in\{\in, q, \in \vee q, \in \wedge q\}$, a contradiction. Hence $F((x a) y)>0$, that is, $(x a) y \in F_{0}$. Consequently, $F_{0}$ is an interior ideal of $S$.

Theorem 4.11. Let I be an interior ideal and $F$ a fuzzy subset of $S$ such that

(1) $(\forall x \in S \backslash I)(F(x)=0)$,

(2) $(\forall x \in I)(F(x) \geq 0.5)$. 
Then

(a) $F$ is a $(q, \in \vee q)$-fuzzy interior ideal of $S$,

(b) $F$ is an $(\in, \in \vee q)$-fuzzy interior ideal of $S$.

Proof. Let $x, y \in S$ and $\lambda_{1}, \lambda_{2} \in(0,1]$ be such that $x_{\lambda_{1}} q F$ and $y_{\lambda_{2}} q F$. Then $x, y \in I$ and we have $x y \in I$. If $\min \left\{\lambda_{1}, \lambda_{2}\right\} \leq 0.5$, then $F(x y) \geq 0.5 \geq \min \left\{\lambda_{1}, \lambda_{2}\right\}$ and hence $(x y)_{\min }\left\{\lambda_{1}, \lambda_{2}\right\} \in F$. If $\min \left\{\lambda_{1}, \lambda_{2}\right\}>0.5$, then

$$
F(x y)+\min \left\{\lambda_{1}, \lambda_{2}\right\}>0.5+0.5=1,
$$

and so $(x y)_{\min \left\{\lambda_{1}, \lambda_{2}\right\}} \mathrm{q} F$. Therefore $(x y)_{\min \left\{\lambda_{1}, \lambda_{2}\right\}} \in \mathrm{vq} F$. Let $x, y, a \in S$ and $\lambda \in(0,1]$ be such that $a_{\lambda_{1}} q F$. Then $a \in I$ and we have $(x a) y \in(S I) S \subseteq I$. If $\lambda_{1} \leq 0.5$, then $F((x a) y) \geq 0.5 \geq \lambda$ and hence $((x a) y)_{\lambda} \in F$. If $\lambda_{1}>0.5$, then

$$
F((x a) y)+\lambda \geq 0.5+0.5=1
$$

and so $((x a) y)_{\lambda} q F$. Therefore $((x a) y)_{\lambda} \in \vee q F$. Therefore $F$ is a $(q, \in \vee q)$-fuzzy interior ideal of $S$.

Let $x, y \in S$ and $\lambda_{1}, \lambda_{2} \in(0,1]$ be such that $x_{\lambda_{1}} \in F$ and $y_{\lambda_{2}} \in F$. Then $x, y \in I$ and we have $x y \in I$. If $\min \left\{\lambda_{1}, \lambda_{2}\right\} \leq 0.5$, then $F(x y) \geq 0.5 \geq \min \left\{\lambda_{1}, \lambda_{2}\right\}$ and hence $(x y)_{\min \left\{\lambda_{1}, \lambda_{2}\right\}} \in F$. If $\min \left\{\lambda_{1}, \lambda_{2}\right\}>0.5$, then

$$
F(x y)+\min \left\{\lambda_{1}, \lambda_{2}\right\}>0.5+0.5=1,
$$

and so $(x y)_{\min \left\{\lambda_{1}, \lambda_{2}\right\}} q F$. Therefore $(x y)_{\min \left\{\lambda_{1}, \lambda_{2}\right\}} \in \vee q F$. Now let $x, y, a \in S$ and $\lambda \in(0,1]$ be such that $a_{\lambda} \in F$. Then $a \in I$ and we have $(x a) y \in I$. If $\lambda \leq 0.5$, then $F((x a) y) \geq 0.5 \geq \lambda$ and hence $((x a) y)_{\lambda} \in F$. If $\lambda>0.5$, then

$$
F((x a) y)+\lambda>0.5+0.5=1,
$$

and so $((x a) y)_{\lambda} q F$. Therefore

$$
((x a) y)_{\curlywedge} \in \vee q F
$$

and so $F$ is an $(\in, \in \vee q)$-fuzzy interior ideal of $S$.

From Example 4.5, we see that an $(\epsilon, \in \vee q)$-fuzzy interior ideal is not a $(q, \in \vee q)$-fuzzy interior ideal (Example 4.5, iv).

In the following theorem we give a condition for an $(\in, \in \vee q)$-fuzzy interior ideal to be an $(\epsilon, \in)$-fuzzy interior ideal of $S$.

Theorem 4.12. Let $F$ be an $(\in, \in \vee q)$-fuzzy interior ideal of $S$ such that $F(x)<0.5$ for all $x \in S$. Then $F$ is an $(\in, \in)$-fuzzy interior ideal of $S$. 
Proof. Let $x, y \in S$ and $\lambda_{1}, \lambda_{2} \in(0,1]$ be such that $x_{\lambda_{1}}, y_{\lambda_{2}} \in F$. Then $F(x) \geq \lambda_{1}$ and $F(y) \geq \lambda_{2}$ and so $F(x y) \geq \min \{F(x), F(y), 0.5\} \geq \min \left\{\lambda_{1}, \lambda_{2}, 0.5\right\}=\min \left\{\lambda_{1}, \lambda_{2}\right\}$ and hence $(x y)_{\min \left\{\lambda_{1}, \lambda_{2}\right\}} \in F$. Now, let $x, y, a \in S$ and $\lambda \in(0,1]$ be such that $a_{\lambda} \in F$. Then $F(a) \geq \lambda$ and we have

$$
F((x a) y) \geq F(a) \geq \lambda
$$

consequently, $((x a) y)_{\lambda} \in F$. Therefore $F$ is an $(\in, \in)$-fuzzy interior ideal of $S$.

For any fuzzy subset $F$ of an $A G$-groupoid $S$ and $\lambda \in(0,1]$, we denote

$$
Q(F ; \lambda):=\left\{x \in S \mid x_{\lambda} q F\right\}, \quad[F]_{\lambda}:=\left\{x \in S \mid x_{\lambda} \in \vee q F\right\} .
$$

Obviously, $[F]_{\lambda}=U(F ; \lambda) \cup Q(F ; \lambda)$.

We call $[F]_{\lambda}$ an $(\in \vee q)$-level interior ideal of $F$ and $Q(F ; \lambda)$ a q-level interior ideal of $F$.

We have given a characterization of $(\in, \in \vee q)$-fuzzy interior ideals by using level subsets (see Proposition 4.2). Now we provide another characterization of $(\in, \in \vee q)$-fuzzy interior ideals by using the set $[F]_{\lambda}$.

Theorem 4.13. Let $S$ be an AG-groupoid and $F$ a fuzzy subset of $S$. Then $A$ is an $(\in, \in \vee \vee q)$-fuzzy interior ideal of $S$ if and only if $[F]_{\lambda}$ is an interior ideal of $S$ for all $\lambda \in(0,1]$.

Proof. Let $F$ be an $(\in, \in \vee q)$-fuzzy interior ideal of $S$. Let $x, y \in[F]_{\lambda}$ for $\lambda \in(0,1]$. Then $x_{\lambda} \in \vee q F$ and $y_{\lambda} \in \vee q F$, that is, $F(x) \geq t$ or $F(x)+t \geq 1$, and $F(y) \geq t$ or $F(y)+t \geq 1$. Since $F$ is an $(\in, \in \vee q)$-fuzzy interior ideal of $S$, we have

$$
F(x y) \geq \min \{F(x), F(y), 0.5\} .
$$

We discuss the following cases.

Case 1. Let $F(x) \geq \lambda$ and $F(y) \geq \lambda$. If $\lambda>0.5$, then

$$
F(x y) \geq \min \{F(x), F(y), 0.5\}=0.5,
$$

and hence $(x y)_{\lambda} \in F$. If $\lambda \leq 0.5$. Then

$$
F(x y) \geq \min \{F(x), F(y), 0.5\} \geq \lambda,
$$

and so $(x y)_{\curlywedge} \in F$. Hence $(x y)_{\lambda} \in \vee q F$.

Case 2. Let $F(x) \geq \lambda$ and $F(y)+\lambda \geq 1$. If $\lambda>0.5$, then

$$
F(x y) \geq \min \{F(x), F(y), 0.5\},
$$

and we discuss the following cases. 
If $\lambda>0.5$, then

$$
\begin{aligned}
F(x y) & \geq \min \{F(x), F(y), 0.5\} \\
& =\min \{F(y), 0.5\} \\
& \geq \min \{1-\lambda, 0.5\}=1-\lambda,
\end{aligned}
$$

that is, $F(x y)+t \geq 1$ and thus $(x y)_{t} q F$. If $\lambda \leq 0.5$, then

$$
F(x y) \geq \min \{F(x), F(y), 0.5\} \geq \min \{\lambda, 1-\lambda, 0.5\}=\lambda,
$$

and so $(x y)_{\lambda} \in F$. Hence $(x y)_{\lambda} \in \vee q F$.

Case 3. Let $F(x)+\lambda \geq 1$ and $F(y) \geq \lambda$. If $\lambda<0.5$, then

$$
F(x y) \geq \min \{F(x), F(y), 0.5\} \geq \min \{F(x), 0.5\} \geq \min \{1-\lambda, 0.5\}=1-\lambda,
$$

that is, $F(x y)+\lambda \geq 1$ and hence $(x y)_{\lambda} q F$. If $\lambda<0.5$, then

$$
\begin{aligned}
F(x y) & \geq \min \{F(x), F(y), 0.5\} \\
& \geq \min \{1-\lambda, \lambda, 0.5\}=\lambda,
\end{aligned}
$$

and so $(x y)_{\lambda} \in F$. Hence $(x y)_{\lambda} \in \vee q F$.

Case 4. Let $F(x)+\lambda \geq 1$ and $F(y)+\lambda \geq 1$. If $\lambda>0.5$, then

$$
F(x y) \geq \min \{F(x), F(y), 0.5\}>\min \{1-\lambda, 0.5\}=1-\lambda,
$$

that is, $F(x y)+\lambda \geq 1$ and thus $(x y)_{\lambda} q F$. If $\lambda \leq 0.5$, then

$$
F(x y) \geq \min \{F(x), F(y), 0.5\} \geq \min \{1-\lambda, 0.5\}=0.5 \geq \lambda,
$$

and so $(x y)_{\lambda} \in F$. Thus in any case, we have $(x y)_{\lambda} \in \vee q F$. Therefore $x y \in[F]_{\lambda}$. Now, let $a \in[F]_{\lambda}$ for $\lambda \in(0,1]$. Then $a_{\lambda} \in \vee q F$, that is, $F(x) \geq \lambda$ or $F(x)+\lambda \geq 1$. Since $F$ is an $(\in, \in \vee q)$-fuzzy interior ideal of $S$, we have

$$
F((x a) y) \geq F(a)
$$

Case 1. Let $F(a) \geq \lambda$. If $\lambda \geq 0.5$, then

$$
F((x a) y) \geq F(a) \geq 0.5
$$


and hence $((x a) y)_{\lambda} q F$. If $\lambda<0.5$, then

$$
F((x a) y) \geq F(a) \geq \lambda
$$

and so $((x a) y)_{\lambda} \in F$. Hence $((x a) y)_{\lambda} \in \vee q F$.

Case 2. Let $F(a) \geq \lambda$ and $F(a)+\lambda \geq 1$. If $\lambda \geq 0.5$, then

$$
F((x a) y) \geq F(a) \lambda \geq 0.5 \text {. }
$$

If $\lambda<0.5$, then

$$
F((x a) y) \geq F(a) \geq \min \{1-\lambda, 0.5\}=1-\lambda,
$$

that is, $F((x a) y)+\lambda \geq 1$ and thus $((x a) y)_{\curlywedge} q F$. If $\lambda \leq 0.5$, then

$$
F((x a) y) \geq F(a) \geq \min \{\lambda, 1-\lambda, 0.5\}=\lambda,
$$

and so $((x a) y)_{\lambda} \in F$. Hence $((x a) y)_{\lambda} \in \vee q A$.

Thus in any case, we have $((x a) y)_{\lambda} \in V q F$. Therefore $(x a) y \in[F]_{\lambda}$.

Conversely, let $F$ be a fuzzy subset of $S$ and let $x, y, a \in S$ be such that $F(x y)<\lambda<\min$ $\{F(x), F(y), 0.5\}$ for some $\lambda \in(0,0.5]$. Then $x, y \in U(F ; \lambda) \subseteq[F]_{\lambda}$, it implies that $x y \in[F]_{\lambda}$. Hence $F(x y) \geq \lambda$ or $F(x y)+\lambda \geq 1$, a contradiction. Hence $F(x y) \geq \min \{F(x), F(y), 0.5\}$ for all $x, y \in S$. Now let $F((x a) y)<F(a)$ for some $x, y, a \in S$. Choose $\lambda$ such that $F((x a) y)<\lambda<$ $F(a)$. Then $a \in U(F ; \lambda) \subseteq[F]_{\lambda}$. It follows that $(x a) y \in[F]_{\lambda}$. This implies that $F((x a) y) \geq \lambda$ or $F((x a) y)+\lambda>1$, a contradiction. Hence $F((x a) y) \geq \min \{F(a), 0.5\}$ for all $x, y, a \in S$. By Proposition 4.2, it follows that $F$ is an $(\in, \in \vee q)$-fuzzy interior ideal of $S$.

$U(F ; \lambda)$ and $[F]_{\lambda}$ are interior ideals of $S$ for all $\lambda \in(0,1]$, but $Q(F ; \lambda)$ is not an interior ideal of $G$ for all $\lambda \in(0,1]$, in general. As shown in the following example.

Example 4.14. Consider the $A G$-groupoid as given in Example 4.5. Define a fuzzy subset $F$ by

$$
F(a)=0.8, \quad F(c)=0.6, \quad F(d)=0.4, \quad F(e)=0.2, \quad F(b)=0.1 .
$$

Then $Q(F ; \lambda)=\{a, c, d\}$ for all $0.2<\lambda \leq 0.4$. Since $c_{0.56} \in F$ and $e_{0.18} \in F$ but $(c e)_{\min \{0.56,0.18\}}=d_{0.18} \bar{q} F$, hence $Q(F ; \lambda)$ is not an interior ideal of $S$ for all $\lambda \in(0.2,0.4]$.

Proposition 4.15. If $\left\{F_{i}\right\}_{i \in I}$ is a family of $(\in, \in \vee q)$-fuzzy bi-ideals of an AG-groupoid $S$, then $\bigcap_{i \in I} F_{i}$ is an $(\in, \in \vee q)$-fuzzy bi-ideal of $S$. 
Proof. Let $\left\{F_{i}\right\}_{i \in I}$ be a family of $(\epsilon, \in \vee q)$-fuzzy bi-ideals of $S$. Let $x, y \in S$. Then

$$
\begin{aligned}
\left(\bigcap_{i \in I} F_{i}\right)(x y) & =\bigwedge_{i \in I} F_{i}(x y) \geq \bigwedge_{i \in I}\left(F_{i}(x) \wedge F_{i}(y)\right) \\
& =\left(\bigwedge_{i \in I} F_{i}(x) \wedge \bigwedge_{i \in I} F_{i}(y)\right) \\
& =\left(\bigcap_{i \in I} F_{i}\right)(x) \wedge\left(\bigcap_{i \in I} F_{i}\right)(y) .
\end{aligned}
$$

Let $x, y, a \in G$. Then

$$
\begin{aligned}
\left(\bigcap_{i \in I} F_{i}\right)((x a) y) & =\bigwedge_{i \in I} F_{i}((x a) y) \geq \bigwedge_{i \in I}\left(F_{i}(a)\right) \\
& =\left(\bigcap_{i \in I} F_{i}\right)(a) .
\end{aligned}
$$

Thus $\bigcap_{i \in I} F_{i}$ is an $(\in, \in \vee q)$-fuzzy interior ideal of $S$.

Definition 4.16. Let $S$ be an $A G$-groupoid and $F$ a fuzzy subset of $S$. Then $F$ is called a strongly fuzzy interior ideal of $S$, if it satisfies the following conditions.

(B9) $(\forall x, y \in S)(F(x y)>\min \{F(x), F(y)\})$.

(B10) $(\forall x, y, a \in S)((F(x a) y)>F(a))$.

Every fuzzy interior ideal of an $A G$-groupoid $S$ is strongly fuzzy interior ideal of $S$.

Theorem 4.17. For any fuzzy subset $F$ of $S$. The conditions (B9) and (B10) of Definition 4.16 are equivalent to the following.

(B11) $(\forall x, y \in S)\left(\forall \lambda_{1}, \lambda_{2} \in(0,1]\right)\left(x_{\lambda_{1}} \in F, y_{\lambda_{2}} \in F \rightarrow(x y)_{\min \left\{\lambda_{1}, \lambda_{2}\right\}} \in F\right)$.

(B12) $(\forall x, y, a \in S)(\forall \lambda \in(0,1])\left(a_{\lambda} \in F \rightarrow((x a) y)_{\lambda} \in F\right)$.

Proof. (B9) $\rightarrow$ (B11). Let $F$ be a fuzzy subset of $S$. Let $x, y \in S$ and $\lambda_{1}, \lambda_{2} \in(0,1]$ be such that $x_{\lambda_{1}} \in F, y_{\lambda_{2}} \in F$. Then $F(x)>\lambda_{1}$ and $F(y)>\lambda_{2}$. Using (B9)

$$
F(x y)>\min \{F(x), F(y)\}>\min \left\{\lambda_{1}, \lambda_{2}\right\},
$$

and so $(x y)_{\min \left\{\lambda_{1}, \lambda_{2}\right\}} \in F$.

$(B 11) \rightarrow(B 9)$. Let $x, y \in S$. Since $x_{F(x)} \in F$ and $y_{F(y)} \in F$. Then by (B9), we have

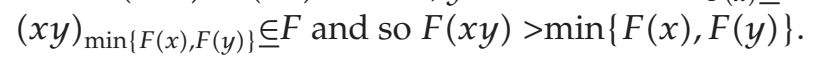



we have

$(B 10) \rightarrow(B 11)$. Let $x, y, a \in S$ and $\lambda \in(0,1]$ be such that $a_{\lambda} \in F$. Then $F(a)>\lambda$. By $(B 10)$

$$
F((x a) y)>F(a)>\lambda,
$$

and so $((x a) y)_{\lambda} \in F$.

$(B 11) \rightarrow(B 10)$. Let $x, y \in S$. Since $a_{F(a)} \in F$, by $(B 11)$, we have $((x a) y)_{F(a)} \in F$ and so $F((x a) y)>F(a)$.

\section{5. $(\underline{\epsilon}, \underline{\in} \vee \underline{q})$-Fuzzy Interior Ideals}

In this section we define the notions of $(\underline{\epsilon}, \underline{\in} \vee q)$-fuzzy interior ideals of an Abel Grassmann's groupoid and investigate some of their properties in terms of $(\underline{\epsilon}, \underline{\in \vee} q)$-fuzzy interior ideals.

Let $F$ be a fuzzy subset of $S$ and $F(x)<0.5$ for all $x \in S$. Let $x \in S$ and $\lambda \in(0,1]$ be such that $x_{\lambda} \underline{\in} \wedge q F$. Then $x_{\lambda} \underline{E F}$ and $x_{\lambda} \underline{q} F$ and so $F(x)>\lambda$ and $F(x)+\lambda>1$. It follows that $1<F(x)+\lambda<\overline{F(x)}+F(x)=2 F(x)$, and so $F(x)>0.5$, which is a contradiction. This means that $\left\{x \in S \mid x_{\curlywedge} \underline{\in} \wedge \underline{q} F\right\}=\emptyset$.

Definition 5.1. A fuzzy subset $F$ of $S$ is called an $(\underline{\alpha}, \underline{\beta})$-fuzzy interior ideal of $S$, where $\alpha \neq \underline{\epsilon} \wedge q$, if it satisfies the following conditions.

(B13) $(\forall x, y \in S)\left(\forall \lambda_{1}, \lambda_{2} \in(0,1]\right)\left(x_{\lambda_{1}} \underline{\alpha} F, y_{\lambda_{2}} \underline{\alpha} F \rightarrow(x y)_{\min \left\{\lambda_{1}, \lambda_{2}\right\}} \underline{\beta} F\right)$.

$(B 14)(\forall x, y, a \in S)(\forall \lambda \in(0,1])\left(a_{\lambda} \underline{\alpha} F \rightarrow((x a) y)_{\lambda} \underline{\beta} F\right)$.

Proposition 5.2. Let $F$ be a fuzzy subset of $S$. If $\underline{\alpha}=\underline{\epsilon}$ and $\beta=\underline{\in} \vee q$ in Definition 5.1. Then (B13), and (B14), respectively, of Definition 5.1, are equivalent to the following conditions.

(B15) $(\forall x, y \in S)(F(x y) \geq \min \{F(x), F(y), 0.5\})$.

(B16) $(\forall x, y, a \in S)(F((x a) y) \geq \min \{F(a), 0.5\})$.

Remark 5.3. A fuzzy subset $F$ of an $A G$-groupoid $S$ is an $(\underline{\epsilon}, \underline{\in} \vee \underline{q})$-fuzzy interior ideal of $S$ if and only if it satisfies conditions (B15) and (B16) of the above proposition.

Using Proposition 5.2, we have the following characterization of $(\underline{\epsilon}, \underline{\in} \vee \underline{q})$-fuzzy interior ideals of an AG-groupoid.

Lemma 5.4. Let $S$ be an $A G$-groupoid and $\emptyset \neq I \subseteq S$. Then $I$ is an interior ideal of $S$ if and only if the characteristic function $X_{I}$ of $I$ is an $(\underline{\in}, \underline{\in} \vee \underline{q})$-fuzzy interior ideal of $S$.

\section{References}

[1] S. K. Bhakat and P. Das, "Fuzzy subrings and ideals redefined," Fuzzy Sets and Systems, vol. 81, no. 3, pp. 383-393, 1996.

[2] S. K. Bhakat and P. Das, "On the definition of a fuzzy subgroup," Fuzzy Sets and Systems, vol. 51, no. 2, pp. 235-241, 1992.

[3] B. Davvaz, "Fuzzy R-subgroups with thresholds of near-rings and implication operators," Soft Computing, vol. 12, no. 9, pp. 875-879, 2008. 
[4] B. Davvaz, " $(\in, \in \vee q)$-fuzzy subnear-rings and ideals," Soft Computing, vol. 10, no. 3, pp. 206-211, 2006.

[5] Y. B. Jun and S. Z. Song, "Generalized fuzzy interior ideals in semigroups," Information Sciences, vol. 176, no. 20, pp. 3079-3093, 2006.

[6] O. Kazanci and S. Yamak, "Generalized fuzzy bi-ideals of semigroups," Soft Computing, vol. 12, no. 11, pp. 1119-1124, 2008.

[7] B. Davvaz, O. Kazanci, and S. Yamak, "Generalized fuzzy n-ary subpolygroups endowed with interval valued membership functions," Journal of Intelligent and Fuzzy Systems, vol. 20, no. 4-5, pp. 159-168, 2009.

[8] B. Davvaz and M. Mozafar, " $(\in, \in \vee q)$-fuzzy Lie subalgebra and ideals," International Journal of Fuzzy Systems, vol. 11, no. 2, pp. 123-129, 2009.

[9] B. Davvaz and P. Corsini, "On $(\alpha, \beta)$-fuzzy $H_{v}$-ideals of $H_{v}$-rings," Iranian Journal of Fuzzy Systems, vol. 5, no. 2, pp. 35-47, 2008

[10] B. Davvaz, J. Zhan, and K. P. Shum, “Generalized fuzzy polygroups endowed with interval valued membership functions," Journal of Intelligent and Fuzzy Systems, vol. 19, no. 3, pp. 181-188, 2008.

[11] O. Kazanc1, B. Davvaz, and S. Yamak, "Fuzzy $n$-ary polygroups related to fuzzy points," Computers $\mathcal{E}$ Mathematics with Applications, vol. 58, no. 7, pp. 1466-1474, 2009.

[12] Y. B. Jun, A. Khan, and M. Shabir, "Ordered semigroups characterized by their $(\in, \in \vee q)$-fuzzy biideals," Bulletin of the Malaysian Mathematical Sciences Society, vol. 32, no. 3, pp. 391-408, 2009.

[13] Y. B. Jun, "On $(\alpha, \beta)$-fuzzy subalgebras of BCK/BCI-algebras," Bulletin of the Korean Mathematical Society, vol. 42, no. 4, pp. 703-711, 2005.

[14] J. Zhan and B. Davvaz, "Generalized fuzzy ideals of near-rings," Applied Mathematics. Journal of Chinese Universities, vol. 24, no. 3, pp. 343-349, 2009.

[15] J. Zhan, B. Davvaz, and K. P. Shum, "A new view of fuzzy hyperquasigroups," Journal of Intelligent and Fuzzy Systems, vol. 20, no. 4-5, pp. 147-157, 2009.

[16] J. Zhan, Y. B. Jun, and B. Davvaz, “On $(\in, \in \vee q)$-fuzzy ideals of BCI-algebras,” Iranian Journal of Fuzzy Systems, vol. 6, no. 1, pp. 81-94, 2009.

[17] Y. B. Jun, "Fuzzy subalgebras of type $(\alpha, \beta)$ in BCK/BCI-algebras," Kyungpook Mathematical Journal, vol. 47, no. 3, pp. 403-410, 2007.

[18] C. Z. Luo, Introduction to Fuzzy Sets I, II, Beijing Normal University Press, Beijing, China, 1989.

[19] P. M. Pu and Y. M. Liu, "Fuzzy topology. I. Neighborhood structure of a fuzzy point and Moore-Smith convergence," Journal of Mathematical Analysis and Applications, vol. 76, no. 2, pp. 571-599, 1980.

[20] M. A. Kazim and M. Naseeruddin, "On almost semigroups," The Aligarh Bulletin of Mathematics, vol. 2, pp. 1-7, 1972.

[21] M. Khan and M. Nouman Aslam Khan, "Fuzzy Abel Grassmann's groupoids," accepted in Advances in Fuzzy Mathematics (AFM), http://arxiv.org/abs/0904.0077. 


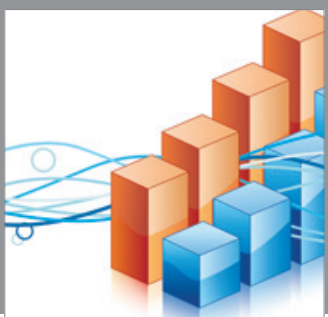

Advances in

Operations Research

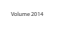

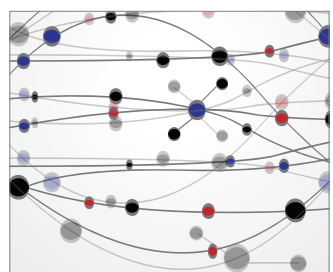

\section{The Scientific} World Journal
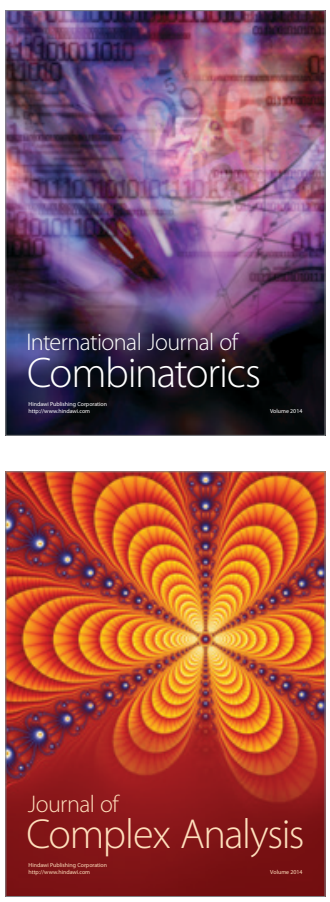

International Journal of

Mathematics and

Mathematical

Sciences
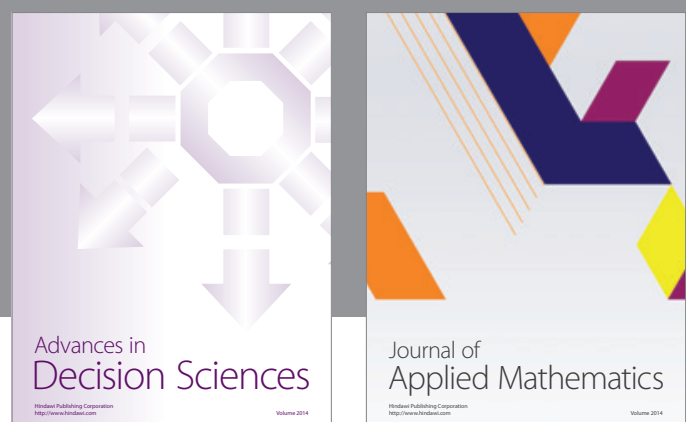

Journal of

Applied Mathematics
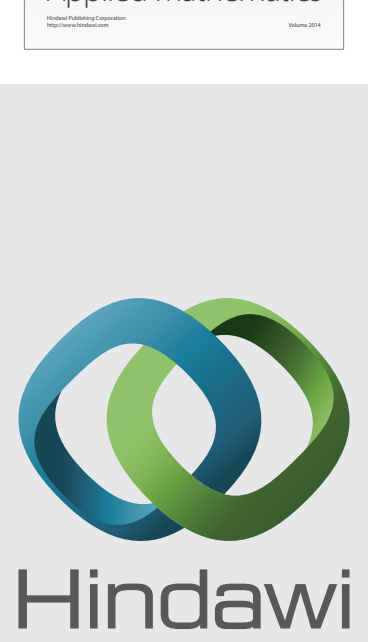

Submit your manuscripts at http://www.hindawi.com
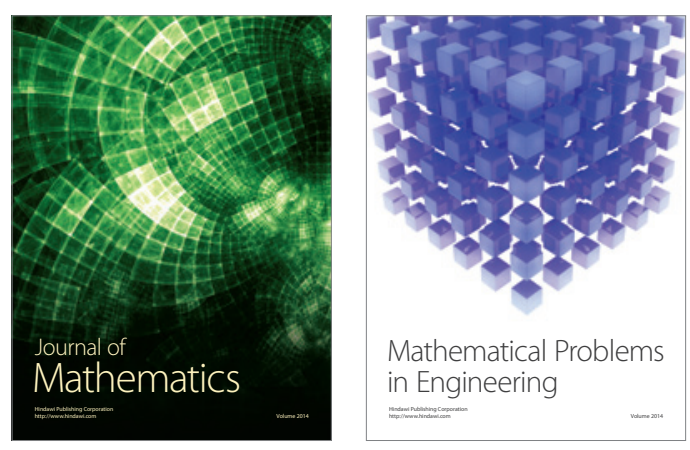

Mathematical Problems in Engineering
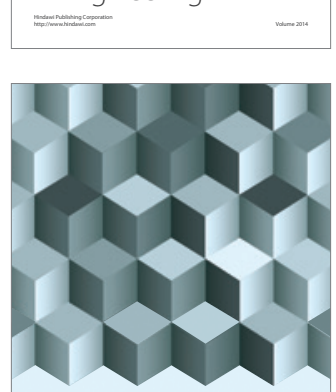

Journal of

Function Spaces
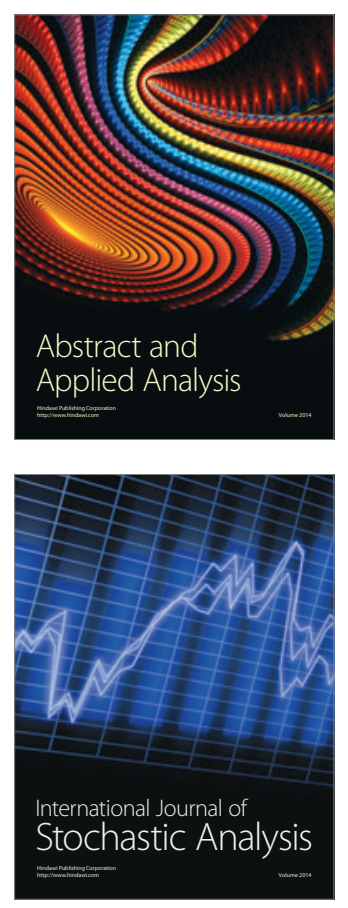

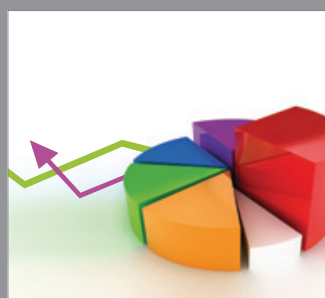

ournal of

Probability and Statistics

Promensencen
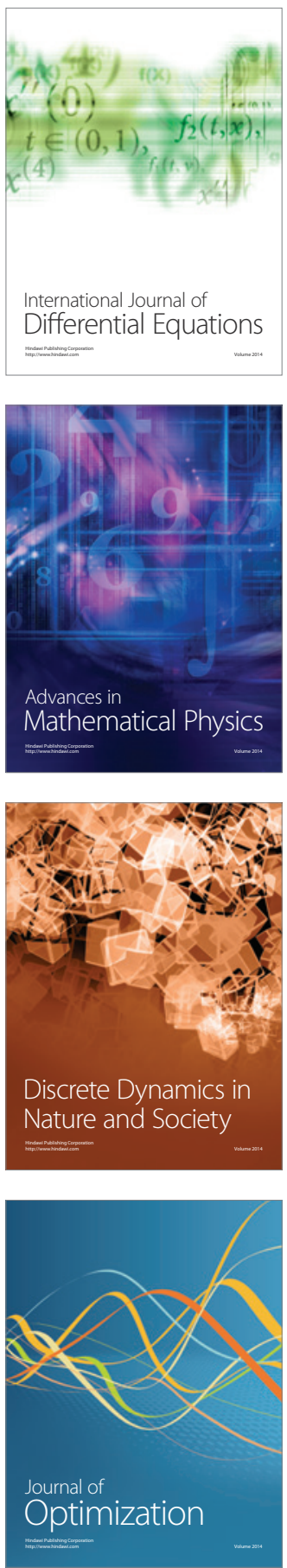